Revue interdisciplinaire des études canadiennes en

France

79 | 2015

Regards croisés Canada-Europe

\title{
La littérature québécoise en Terre d'Amérique : points de vue français et allemands
}

Quebecois literature in America: French and German viewpoints in literary anthologies

\section{Sophie Dubois}

\section{(2) OpenEdition}

Journals

Édition électronique

URL : http://journals.openedition.org/eccs/578

DOI : $10.4000 /$ eccs.578

ISSN : 2429-4667

Éditeur

Association française des études canadiennes (AFEC)

Édition imprimée

Date de publication : 1 décembre 2015

Pagination : 49-69

ISSN : 0153-1700

Référence électronique

Sophie Dubois, "La littérature québécoise en Terre d'Amérique : points de vue français et allemands », Études canadiennes / Canadian Studies [En ligne], 79 | 2015, mis en ligne le 01 décembre 2016, consulté le 30 avril 2019. URL : http://journals.openedition.org/eccs/578 ; DOI : 10.4000/eccs.578 


\section{LA LITTÉRATURE QUÉBÉCOISE EN TERRE D'AMÉRIQUE : POINTS DE VUE FRANÇAIS ET ALLEMANDS}

\section{Sophie Dubois}

Université de la Sarre

Le présent article ${ }^{1}$ étudie l'ancrage américain de la littérature québécoise dans quatre anthologies ou manuels sur le Québec, parus en France et en Allemagne. À travers l'analyse de la représentation du mythe américain et du statut octroyé à la langue française, nous comparons les perceptions des deux pays européens. Au final, nous nous interrogeons sur la pertinence et sur les conséquences d'une telle inscription de la littérature québécoise et nous examinons ce qu'un tel choix révèle des préoccupations respectives des deux pays.

This paper examines four anthologies or textbooks on Quebec published in France and in Germany that sets Quebec literature in an American context. Through an analysis of the representation of the American myth and of the status given to the French language, we compare the perceptions of these two European countries. Finally, we question the relevance and the consequences of such an anchoring of Quebec literature and we investigate what such a choice reveals about the respective concerns of these two countries.

La littérature québécoise a aujourd'hui des lecteurs à travers le monde et elle occupe une place dans les cursus scolaires de plusieurs pays s'intéressant à la francophonie. Le plus souvent, les anthologies et les manuels parus à l'étranger incluent donc la littérature québécoise dans l'espace des pays francophones. Or, d'autres ouvrages récents tendent plutôt à en faire une littérature d'Amérique. Si cette inscription est courante - et de plus en plus assumée - au Québec, comme l'ont notamment montré les travaux de Jean Morency (1994), elle prend une signification différente et se charge d'implications nouvelles lorsqu'elle est posée par un regard extérieur.

Ancrée dans une perspective de perception interculturelle, cet article vise à interroger l'ancrage américain de la littérature québécoise à l'étranger, à partir de l'analyse d'un manuel et de trois anthologies parus en France et en Allemagne, et à comparer la manière dont les deux pays posent et justifient cette inscription. Après une brève présentation du corpus, accompagnée de considérations théoriques concernant le choix des objets d'étude, j'étudierai, de façon comparative, la représentation du mythe américain et le statut octroyé à la langue française dans les ouvrages français et allemands. Enfin, j'aborderai la

\footnotetext{
${ }^{1}$ Cette recherche a été effectuée dans le cadre d'un postdoctorat financé par le CRSH (Conseil de recherche en sciences humaines) du Canada.
} 


\section{Sophie DUBOIS}

pertinence de l'ancrage américain de la littérature québécoise et les conséquences de celui-ci sur son appréhension et sa compréhension à l'étranger.

De façon plus large, cette étude a pour but de déceler ce que le choix d'une telle inscription révèle des préoccupations respectives des deux pays européens car, pour paraphraser un postulat clé de l'imagologie en littérature comparée, parler des autres, c'est toujours aussi parler de soi. (voir, entre autres, les travaux de Daniel-Henri Pageaux $(1995,2007))$

\section{Les manuels et anthologies de littérature étrangère}

Bien qu'ayant des visées souvent différentes, les genres du manuel et de l'anthologie littéraire ont en commun de proposer une sélection, une organisation, et - de façon plus ou moins explicite - une interprétation de la production d'une communauté ou d'une aire culturelle. En ce sens, ils apparaissent comme des formes privilégiées lorsqu'il s'agit de présenter une littérature étrangère à un public non initié. Ces ouvrages se prêtent bien en effet «à un regard d'ensemble que l'ampleur de la production et la méconnaissance de celle-ci par les lecteurs [...] peuvent rendre malaisé en l'absence de sélection ${ }^{2} »$ (FRAISSE 1997: 108). Échantillon et condensé de la littérature d'un pays, les anthologies et manuels parus à l'étranger jouent le rôle de médiateurs culturels offrant aux lecteurs de la culture cible, certaines clés pour comprendre la culture source et sa littérature. Aussi rendent-ils cette littérature non seulement accessible, mais également cohérente et pertinente pour ses lecteurs étrangers. Ils se révèlent, de ce fait, à la fois emblématiques de ce qui, dans la culture d'origine, est jugé digne de transmission et de ce qui est susceptible d'intéresser la culture d'accueil. À travers leur regard sur l'Autre, ces ouvrages dévoilent donc, du même souffle, les préoccupations de leur pays respectif. En effet, même s'il a pour but de transmettre des connaissances sur une culture ou une littérature étrangère, le manuel (mais cela vaut aussi pour l'anthologie) demeure « un puissant vecteur d'idéologie et de culture dont on s'accorde à penser [...] qu'il participe étroitement de la construction identitaire et de la formation des mentalités collectives » (CHOPPIN 2007 : 111). Il renseigne donc tout autant sur la culture source que sur les valeurs et les goûts esthétiques privilégiés par la culture cible. Dans cette optique, les quatre ouvrages du corpus fournissent des lieux pertinents pour étudier - et comparer - les

\footnotetext{
${ }^{2}$ Les propos de Fraisse concernent d'abord la forme anthologique. Or, il mentionne bien que sa définition de l'anthologie ne repose pas sur l'usage qui est fait de l'ouvrage, mais sur des critères internes et littéraires qui peuvent inclure la forme du manuel. (FRAISSE 1997 : 93-98.)
} 


\section{LA LITTÉRATURE QUÉBÉCOISE EN TERRE D’AMÉRIQUE}

perceptions française et allemande de la littérature québécoise, par le biais de son inclusion dans le contexte américain.

Le corpus étudié comprend deux anthologies françaises et deux ouvrages (une anthologie et un manuel) allemands. Ceux-ci ont été retenus en fonction de deux critères : il s'agit d'ouvrages récents inscrivant, à même leur titre, la littérature québécoise (ou plus largement le Québec) dans l'Amérique.

Du côté français, le plus ancien des ouvrages est l'anthologie Québec-Acadie. Rêves d'Amérique ${ }^{3}$, parue en 1998, aux éditions Omnibus. Dirigée par JeanPaul Bouchon, Alain Quella-Villéger et Dominique-Anne Villégier, cette volumineuse anthologie comprend onze romans ou récits ${ }^{4}$ qui témoignent à la fois de l'intérêt des compilateurs pour l'exotisme et les récits de voyage ${ }^{5}$, et de la dimension pittoresque privilégiée par la collection «Une autre façon de voyager » dans laquelle elle est incluse. Publié 10 ans plus tard, en 2008, dans le cadre de la commémoration du $400^{\mathrm{e}}$ anniversaire de la ville de Québec, le deuxième ouvrage français, Amérique, Amériques! Écrits du Québec $1608 / 2008^{6}$, fait également place à l'imaginaire des récits de voyage. Coéditée par L'instant même au Québec et par La Passe du vent en France, cette anthologie paraît dans la collection "Haute mémoire» qui réunit et fait dialoguer des textes du passé et des textes inédits d'auteurs contemporains. Ainsi, dans Amérique, Amériques!, des auteurs comme Claude Beausoleil, Carole David, Jean-Marc Dalpé, Yolande Villemaire ou Hélène Dorion proposent leur vision de l'Amérique en échos à celles d'explorateurs de la Nouvelle-France comme Jacques Cartier, Samuel de Champlain, Marc Lescarbot, Gabriel Sagard ou Paul Lejeune.

Du côté allemand, l'anthologie Anders schreibendes Amerika. Eine Anthologie der Literatur aus Québec 1945-20007, parue en 2000 chez Wunderhorn - une

\footnotetext{
${ }^{3}$ Les références à cet ouvrage se feront, dans le corps du texte, à l'aide de l'abréviation $Q A$.

${ }^{4}$ Léo-Paul Desrosiers, Les Engagés du Grand Portage, 1938 ; Louis Hémon, Maria Chapdelaine, 1914 ; Félix-Antoine Savard, Menaud, maître draveur, 1937 ; Maurice Constantin-Weyer, Un homme se penche sur son passé, 1928 ; Henri Queffélec, Crabes, crevettes, naufrages..., 1974 ; Marie Le Franc, Pêcheurs de Gaspésie, 1938; Maurice Genevoix, Le Lac Fou, 1942 et Eva Charlebois, 1944 ; Yves Thériault, La Mariouche, c'est pour un Blanc, 1960 ; Harry Bernard, Les jours sont longs, 1951, et Henri Longfellow, Évangeline (trad. de Pamphile LeMay), 1912 [1847].

${ }^{5}$ Jean-Paul Bouchon est l'auteur d'ouvrages tels L'Exotisme au féminin et Aventuriers extraordinaires de l'Ouest, alors que Alain Quella-Villégier est historien et spécialiste des récits d'exploration ; il a notamment fondé et dirigé, de 1990 à 2006, la revue Les Carnets de l'Exotisme.

${ }^{6}$ Les références à cet ouvrage se feront, dans le corps du texte, à l'aide de l'abréviation AA.

${ }^{7}$ Les références à cet ouvrage se feront, dans le corps du texte, à l'aide de l'abréviation AsA.
} 


\section{Sophie DUBOIS}

maison d'édition qui s'intéresse à la «périphérie ${ }^{8} »-$, est le résultat de la collaboration entre Lothar Baier, critique et traducteur allemand, et Pierre Filion, auteur et éditeur québécois, qui se sont adjoint Marie-Andrée Lamontagne, Gilbert David et Heinz Weinmann pour les sections portant sur la poésie, le théâtre et l'essai. Organisée en fonction des genres littéraires, elle regroupe des extraits de textes québécois modernes ${ }^{9}$ traduits en allemand. Enfin, le manuel Découvrir le Québec. Une Amérique qui parle français ${ }^{10}$ s'adresse aux classes avancées de français langue étrangère des lycées allemands. Il est à la fois le produit des recherches - et de l'intérêt pour le Québec - de sa rédactrice allemande Helga Bories-Sawala et la réponse à un besoin pédagogique lié à l'ajout du Québec au programme obligatoire de certains Länder en vue de l'Abitur de français (examen terminal équivalant au baccalauréat en France). Outre sa dimension didactique, ce manuel a donc pour particularité de porter sur le Québec en général, tout en faisant une large place à la littérature contemporaine puisque les textes au programme sont d'abord des textes littéraires ${ }^{11}$.

Cette brève présentation des ouvrages à l'étude témoigne de leur diversité. Ainsi, malgré une certaine contemporanéité et une même inscription américaine de la littérature québécoise, ils offrent des formes, des contenus, des visées et des points de vue différents. Ce double rapport de socle commun et de divergences a pour avantage de permettre à la fois la comparaison des ouvrages et une certaine généralisation des observations, découlant d'une perception similaire, relevant d'un même pays. En effet, comme le montreront les analyses qui suivent, les ouvrages français et allemands proposent des regards fort

\footnotetext{
${ }^{8}$ Elle affiche, en exergue de son descriptif: «Die Erneuerung der Literatur kommt aus den Peripherien und nicht aus den Metropolen» («Verlag», Wunderhorn [En ligne] http://www.wunderhorn.de/content/verlag/index ger.html). (« Le renouvellement de la littérature vient de la périphérie et non des métropoles. » (Je traduis.))

${ }^{9}$ Parmi la quarantaine d'auteurs cités, on trouve par exemple Hubert Aquin, Marie-Claire Blais, Yin Chen, Jacques Brault, Paul-Marie Lapointe, Gaston Miron, Michel Marc Bouchard, Normand Chaurette, Marcel Dubé, Claude Gauvreau, Paul-Émile Borduas, Charles Taylor et Pierre Vadeboncœur. Seul Hector de Saint-Denys Garneau ne respecte pas la borne temporelle annoncée par les compilateurs de l'anthologie.

${ }^{10}$ Les références à cet ouvrage, dans le corps du texte, se feront à l'aide des abréviations DQ ou DQ-U lorsqu'elles renvoient au livre du professeur (Unterrichtsmodell).

${ }^{11}$ Il s'agit de quatre nouvelles de Monique Proulx tirées de son recueil Les aurores montréales ( " Oui or no », " Rouge et blanc », " Gris et blanc » et "Jaune et blanc»), de la nouvelle "Un French » de Yannick Jaulin et du conte « Le petit Indien et l'érable » de Jean Muzi. Le programme obligatoire compte également les chansons «Les maudits Français » et «Bleu» de Lynda Lemay, et quelques planches de la bande dessinée «Les nouvelles aventures de Lucky Luke : La belle province $»$.
} 


\section{LA LITTÉRATURE QUÉBÉCOISE EN TERRE D’AMÉRIQUE}

différents tant sur la nature "américaine » du Québec que sur le statut de la langue française en contexte américain.

\section{Le mythe de l'Amérique}

Depuis sa découverte, l'Amérique fascine. Par l'image de l'espace vierge qu'elle offrait aux Européens à leur arrivée, elle incarna l'esprit de renouveau qui anima cette époque, baptisée significativement « Renaissance ». Comme l'a bien montré Jean Morency, le mythe du recommencement, de la transformation initiatique, inspiré par l'expérience de l'espace américain a fortement informé la littérature du continent - aussi bien celle des États-Unis que celle du Québec (MORENCY 1994: 13-18). Mais, comment ce mythe s'exprime-t-il sous le regard de l'étranger envisageant la littérature québécoise ? Quels aspects en sont retenus dans les manuels et anthologies françaises et allemandes?

\section{Le point de vue français : la colonisation française en Amérique}

Les collections dans lesquelles paraissent les deux ouvrages français (« Haute mémoire » et «Une autre façon de voyager ») attestent bien l'inscription de la littérature québécoise dans un cadre qui relève à la fois de l'exploration géographique et du voyage dans le temps, soit celui de la colonisation française en Amérique.

Les deux anthologies font en effet débuter leur récit, non pas avec Jacques Cartier, comme on pourrait s'y attendre dans un ouvrage sur le Québec, mais avec Giovanni da Verrazzano envoyé en Amérique par François $1^{\mathrm{er}}$, et premier explorateur à « relat[er] la découverte de la partie septentrionale du continent » $(A A: 41)$ et à "donne[r] aux rives de Terre-Neuve le nom de "NouvelleFrance" " $(Q A:$ XVII). La découverte du continent américain a donc ici préséance sur celle du Québec. Ces citations révèlent en outre que, bien davantage que par le biais de l'histoire, c'est par celui de la mémoire - laquelle passe par le récit (le fait de relater) et par la trace (celle du toponyme) - que s'établit le lien entre le Québec et la France dans ces anthologies. La raison en est simple : l'histoire de la colonisation française en Amérique (à tout le moins en ce qui concerne le Québec) conduit à une impasse, celle de la cession de la Nouvelle-France à l'Angleterre. Il est en cela révélateur que, dans les deux ouvrages, cet événement disruptif soit éludé. Le télescopage des deux dates (1608/2008) que propose Amérique, Amériques! a effectivement pour conséquence d'évacuer la profondeur historique et, par le fait même, d'occulter la Conquête anglaise afin de maintenir l'illusion d'une Amérique française. De 


\section{Sophie DUBOIS}

même, dans Québec-Acadie, la Conquête est euphémisée, voire récusée : elle est présentée comme le fruit «des rivalités européennes, doublées du soulèvement de nombreuses tribus amérindiennes [au cours desquels] des "arpents" deviennent anglais ou français sans trop savoir comment. » ( $Q A$ : IV. Je souligne.) Les auteurs, en accentuant, d'un côté, la part de blâme dévolue aux Amérindiens et en laissant, d'un autre côté, au hasard ou au destin la responsabilité des cessions de territoire, minimisent le rôle de la France dans la perte de ses colonies américaines. Ainsi délestée du poids de 1760, les œuvres littéraires retenues dans les ouvrages français sont celles qui rappellent l'attachement du Québec à la France, soit, certes, les récits des explorateurs de la Nouvelle-France, mais aussi les romans qui présentent comme thématiques la défense de l'héritage français et le souvenir de ces ancêtres qui ont "marqué un pan du continent nouveau », pour reprendre un passage de Maria Chapdelaine - devenu leitmotiv dans Menaud, maître draveur - qui inscrit d'emblée le Canada français dans une perspective continentale.

L'Amérique dépeinte dans ces deux ouvrages est donc celle des découvreurs, des explorateurs, des missionnaires et, plus tard, des draveurs, coureurs des bois et autres colonisateurs qui ont parcouru et nommé - au nom de la France - le territoire américain. Ainsi, c'est bien dans l'espace, ou plutôt dans le souvenir de l'espace parcouru, que se déploie ici l'image de l'Amérique, dans cette géographie qui, par les « mille noms que des paysans pieux venus de France ont donnés aux lacs, aux rivières et au village de la contrée nouvelle» $(Q A: 284)$, rappelle le passage du temps et le chemin des premiers explorateurs :

Il y avait, raconte le narrateur de Queffélec naviguant sur le fleuve Saint-Laurent, entre [la côte] et la grande île d'Anticosti, le passage reconnu trois siècles plus tôt par Jacques Cartier, large de dix-sept à dixhuit milles et qui portait le nom de l'Explorateur ( $Q A: 516)$.

« De Péribonka à l'Athabaska », suivant le titre de l'introduction de QuébecAcadie, les récits nous transportent, bien au-delà du seul Québec ou même de l'Acadie, le long de la côte est américaine, puis dans le Canada entier, à travers les Prairies (« À Winnipeg, à Brandon, à Régina, à Moose Jaw, à Medecine Hat, à Calgary, à Edmonton, à Prince Albert, à Saskatoon » $(Q A: 445))$ jusqu'aux Rocheuses ou jusqu'aux «plaines du centre américain » $(Q A: 114)$. Cette traversée du territoire donne alors lieu à l'évocation de traditions, d'héritages et de savoir-faire que possédaient les ancêtres français. À travers le mythe typique de l'Amérique des grands espaces et de la nature sauvage à apprivoiser, sont ainsi évoquées la traite des fourrures avec les Amérindiens 


\section{LA LITTÉRATURE QUÉBÉCOISE EN TERRE D’AMÉRIQUE}

(Les Engagés du Grand Portage), la vie dans les forêts du Nord québécois (Les jours sont longs), la pêche dans les Maritimes (Pêcheurs de Gaspésie) ou la villégiature dans les Rocheuses (Eva Charlebois).

Les romans d'aventure ou du terroir composant Québec-Acadie font ainsi écho aux récits de voyage contenus dans Amérique, Amériques!, alors que la contemplation des paysages apparaît comme "l'émouvante reconstitution de notre histoire canadienne » $(Q A: 496)$.

\section{Le mythe : une perspective européenne}

L'évocation fréquente de toponymes dans les deux anthologies françaises représente bien une des spécificités des textes contenus dans ces ouvrages, soit l'importance du langage - de la dénomination - dans l'acte de prise de possession du territoire et, par extension, celle du récit dans la constitution du mythe américain des grands espaces à apprivoiser. Les directeurs des anthologies se montrent d'ailleurs conscients de la part de fiction qui sous-tend cette représentation du Québec. Aussi, tout en y adhérant partiellement et en le reconduisant, cherchent-ils à dévoiler, dans le paratexte des ouvrages, les assises de ce mythe et ses implications sur la perception du Québec et de sa littérature.

Dans son introduction à Amérique, Amériques!, Gilles Pellerin, directeur littéraire à l'Instant Même, met par exemple en cause la pertinence de la paraphrase « Nouveau Monde » pour décrire l'Amérique, lequel n'est en réalité «nouveau » qu'en fonction de la perspective européenne. De son angle de Québécois, il soutient en effet que les Européens n'ont pas «découvert» l'Amérique, mais l'ont «recouvert» $(A A: 40)$ d'un «filtre» $(A A: 33)$, imposant ainsi leur point de vue et leur représentation. De même, les compilateurs de Québec-Acadie, dont la visée explicite est de «di[re] l'épopée d'une nation farouche et vigoureuse » $(Q A: \mathrm{XIII})$, mettent en évidence la tradition littéraire - celle des récits de voyage et des romans d'aventures - sur laquelle repose cette vision épique de l'Amérique. Les intertextes renvoyant à James Fenimore Cooper, Jack London, Alexandre Dumas, Jules Verne ou Chateaubriand, ou les allusions aux personnages de Robinson Crusoé, de Croc blanc ou de Gulliver, apparaissent dès lors comme autant de signes d'un mode d'appréhension de l'Amérique qui passe d'abord par la littérature. De même, les nombreux effets d'échos créés par la présence, dans les premiers récits de l'anthologie, du roman Maria Chapdelaine - ce « livre [qui] disait vrai » ( $Q A$ : 301), selon le personnage de Menaud - révèlent (s'il en était encore besoin) 


\section{Sophie DUBOIS}

l'impact, presque documentaire, qu'a eu ce roman sur la perception du Québec, en France particulièrement.

En somme, dans ces deux ouvrages, le mythe de l'Amérique, des « espaces infinis et lointains » $(Q A:$ III), demeure très présent, ne serait-ce que par le choix des textes compilés. On y décèle néanmoins deux particularités. D'abord, le mythe est fortement ancré dans le contexte de la colonisation française : revendiquant l'existence d'une "véritable société coloniale nord-américaine » ( $Q A: \mathrm{IV})$, les compilateurs tiennent à rappeler que « le Canada des cabanes ce fut d'abord celui des Français » ( $Q A$ : III). Par ailleurs, il est accompagné d'un paratexte révélant la part de médiation - européenne et littéraire - ayant contribué à la formation de l'imaginaire de l'Amérique comme monde " nouveau », vierge, à conquérir et à habiter. Grâce à cette conscience du mythe, celui-ci ne se confond plus avec la réalité - la "Cabane au Canada » appartient bien désormais aux « rêves d'Amérique ».

\section{8 : Persistance et renouvellement du mythe}

Qu'en est-il, dans cette perspective, des textes datant de 2008 réunis dans l'anthologie Amérique, Amériques! ? Écrits expressément pour l'anthologie qui souhaite faire dialoguer des textes des deux époques, ils s'inspirent naturellement de l'imaginaire de la Nouvelle-France. Ces poèmes et ces courts récits évoquent en effet l'image de l'Amérique comme une terre à la fois «neuve [et] ancestrale » $(A A: 159)$, terre à parcourir « de la Rivière Rouge à l'Alberta », comme l'évoque un poème de Jean-Marc Dalpé (AA: 173). À nouveau ici, le territoire physique fait office d'histoire et de filiation: "Je suis de paysage [sic] plutôt que de parents », écrit par exemple Catherine Lalonde $(A A: 241)$. La géographie renvoie ainsi aux origines, imprégnant l'image de l'Amérique d'une nostalgie de la «pureté originelle» $(A A: 19)$, celle de l' "Amérique d'avant l'Amérique », selon la formule de Claude Beausoleil $(A A: 164)$, c'est-à-dire d'avant l'expansion de la culture américaine associée aux États-Unis. Se superpose alors au mythe de l'Amérique originelle, un contre-mythe correspondant à la perception plus actuelle d'une Amérique capitaliste et artificielle, faite d' " envie et [de] culpabilité » $(A A: 210)$, d'une Amérique de bungalows, de repas sous vide et de Toys " $R$ " US (AA:167-169), selon les textes de Isabelle Courteau et de Carole David. En ce sens, les textes contemporains travaillent eux aussi à défaire le mythe de l'Amérique des grands espaces en en révélant l'aspect illusoire. Or, cette mise en cause de l'utopie américaine se fait au prix de la constitution d'un nouveau mythe, aux accents dystopiques cette fois, sur lequel les auteurs ne portent cependant pas 


\section{LA LITTÉRATURE QUÉBÉCOISE EN TERRE D’AMÉRIQUE}

un regard aussi distancié. Comme le laisse entrevoir un poème de Richard Desgagné, l'ancienne représentation - celle des « trappeurs/ cowboys/ chevaux dans la prairie/ la pampa»-, désormais envisagée comme «mythes et illusions ", est remplacée par une nouvelle perception négative et désillusionnée - celle des « gratte-ciel/ [et de] la réussite /[...]/ les Amériques factices/[...]/ les Amériques arènes dénaturées »-qui abolit, au final, toute possibilité d'espoir : « quel rêve ? Quelle utopie? » $(A A: 194)$.

\section{Le point de vue allemand : une terre de possibilités}

C'est justement de ces rêves et de ces utopies dont l'existence est mise en doute dans les anthologies françaises que se réclament les ouvrages allemands qui voient au contraire dans l'Amérique une terre de possibilités, un espace de fantaisie : " einen Raum der Phantasie » (AsA : 11). L'Amérique apparaît, dans l'anthologie Anders schreibendes Amerika et dans le manuel Découvrir le Québec, comme un «nouveau monde», qui n'est toutefois plus celui des explorateurs des $\mathrm{XVII}^{\mathrm{e}}$ et XVIII ${ }^{\mathrm{e}}$ siècles, mais celui de l'époque contemporaine qui s'amorce au lendemain de la Deuxième Guerre mondiale. Les deux ouvrages allemands privilégient en effet une représentation actuelle du Québec et de l'Amérique qui y est dépeinte comme un monde jeune, dont le mythe, selon Baier et Filion, serait encore à construire ${ }^{12}$.

Dans cette perspective, la littérature québécoise est reconnue pour sa vitalité, son caractère exploratoire, sa faculté d'écrire l'Amérique autrement comme l'indique le titre de l'anthologie, c'est-à-dire en dehors du point de vue imposé par les colonisateurs européens que les anthologies françaises - bien qu'elles cherchent à la révéler - reprennent malgré tout. Non confondue avec les ÉtatsUnis, l'Amérique s'oppose plutôt ici à l'espace européen, dont la culture et la répartition sociale seraient figées par le poids des traditions. À l'inverse, l'Amérique y incarne une terre de liberté et de possibilités - celle de l'American Dream en fonction duquel un immigrant peut trouver le «chemin de la richesse ", selon un leitmotiv d'une nouvelle de Monique Proulx ( $D Q$ : 144-145) et un vendeur de hot-dog comme François Galarneau, peut aspirer à devenir écrivain (AsA : 63-69).

${ }^{12}$ « [einem] neuen Welt, deren Mythen noch zu begründen sind » (AsA : 16). 


\section{Sophie DUBOIS}

\section{L'Amérique plurielle}

Cette Amérique de tous les possibles se révèle alors - et le Québec avec elle comme un lieu d'ouverture, de diversité, de pluralité et, on le verra, de polyphonie. Ainsi «perçu sous l'angle de son potentiel interculturel» (MORENCY $2013: 110$ ), le territoire américain offre un terrain privilégié pour la mise en scène de contacts entre différentes cultures.

Les relations interculturelles sont en effet omniprésentes dans les ouvrages des deux pays. Or, si l'Amérique apparaît, dans les anthologies françaises, comme une terre de rencontre et d'échange (entre Amérindiens, Français, Anglais, mais aussi Irlandais et Écossais), le contact avec l'Autre se fait de façon antagonique et n'est souvent qu'une occasion de réaffirmer l'attachement à la tradition française : l'amour et le mariage exogames y sont par exemple condamnés au profit du maintien de la «race» canadienne-française. À l'inverse, dans les anthologies allemandes, les relations entre les «Gens du pays » $(D Q: 131)$ (francophones, anglophones, autochtones et communautés culturelles (incluant les Allemands $\left.{ }^{13}\right)$ ) visent à présenter la diversité du Québec et son identité - non plus strictement française, mais plurielle. Les nouvelles tirées du recueil de Monique Proulx Les aurores montréales (1996) retenues dans le manuel sont en ce sens représentatives du choix du Québec de «l'interculturalisme, comme formule de compromis entre l'assimilation et la ghettoïsation " (Gérard Bouchard, cité dans $D Q: 100)$ : elles mettent en scène, avec humour, les perceptions mutuelles (différences, idées reçues et malentendus interculturels) qui existent entre les Québécois («Blancs»), les autochtones («Rouges») et les immigrants chinois («Jaunes»), italiens («Roses»), sud-américains ( «Gris ») et haïtiens ( "Noirs »). De même, Baier et Filion, dans l'anthologie, soutiennent que le Québec est une des sociétés les plus accueillantes («aufnahmebereiten», $A s A: 15)$ du monde occidental et font valoir l'apport des écrivains migrants à la littérature québécoise: la contribution de ces auteurs, affirment-ils, est de grande importance, en cela qu'ils apportent une représentation du monde différente et qu'ils participent à une conscience moderne tournée vers le monde (AsA: 18). La présence d'écrivains ou de narrateurs migrants dans les deux ouvrages permet en effet de proposer un regard de l'extérieur sur le Québec, ceux-ci ayant pour tâche d'observer les gens d'ici (« die Leute hier zu beobachten »), tout en interrogeant leur propre

\footnotetext{
${ }^{13}$ Bories-Sawala consacre une section de son manuel aux «Allemands du Québec » (DQ : 151153), alors que l'anthologie contient quelques textes qui réfèrent à l'Allemagne, les plus explicites étant les Historiettes de Jacques Ferron (AsA : 56-62) qui évoquent la première vague d'immigration allemande sur les rives du Saint-Laurent lors de la Révolution américaine.
} 


\section{LA LITTÉRATURE QUÉBÉCOISE EN TERRE D’AMÉRIQUE}

identité d'étranger («Identität als Fremder »), comme le souligne le personnage de Sergio Kokis, qui en vient à conclure qu'on est toujours l'étranger (ou le nègre) de quelqu'un d'autre (AsA : 84-85, 89), universalisant ainsi la situation de l'exil, de la migrance et de l'altérité.

\section{L'Amérique universelle}

L'ancrage américain, par l'élargissement du territoire réel et imaginaire d'appartenance qu'il permet, s'avère ainsi un moyen d'universaliser la littérature québécoise et de sortir des questions liées aux tensions entre le Québec et le reste du Canada et au rapport de la province avec la France. Les textes retenus dans les anthologies allemandes donnent à voir un Québec ouvert (ou voulant s'ouvrir) sur le monde : " Dans l'espoir à la surface du globe d'une fissure, / Dans l'espoir d'un éclatement des bornes » (AsA : 203), écrit par exemple Saint-Denys Garneau. Mais c'est Gaston Miron qui paradoxalement exprime le mieux cette ouverture : le grand poète national sait en effet donner à l'imagerie québécoise une portée universelle par des images telles la « ceinture fléchée d'univers » ou la «danse carrée des quatre coins d'horizon» (AsA: 190).

Les ouvrages allemands ne mettent donc pas en scène une exploration du territoire américain, mais une ouverture de l'espace - culturel et géographique - au-delà des frontières du Québec. Dans le même ordre d'idées, les auteurs des ouvrages soulignent les parallèles sociohistoriques qui existent entre le Québec et les mouvements de libération dans le Tiers Monde et les luttes des minorités $(A s A: 10)$ : s'instaure dès lors une solidarité avec les "hommes de peine » (Miron), les opprimés de « tous les séminaires du globe » («Refus global ») ou les « abénaki maya nègre de birmingham ${ }^{14}{ }$ (Paul-Marie Lapointe) (AsA : 195, 295, 170). Bref, l'ancrage américain de la littérature québécoise proposé par les ouvrages allemands permet d'inscrire le Québec dans un monde "plus ouvert » où « les cousins se sont éloignés » $(D Q: 12)$, écrit Yannick Jaulin dans son texte «Un French». Au final, il contribue à la rendre pertinente pour le public allemand qui n'entretient pas d'emblée un lien d'attachement historique ou sentimental avec le Québec, comme c'est le cas de la France.

En somme, alors que l'inclusion de la littérature québécoise dans le continent américain sert, du côté français, à une réinscription de la filiation entre la France et le Québec par le biais du passé colonial, il permet, du côté allemand,

\footnotetext{
${ }^{14}$ Cette référence renverrait à Martin Luther King, emprisonné à Birmingham en 1963.
} 


\section{Sophie DUBOIS}

de rompre ce lien par-delà lequel la littérature québécoise acquiert une dimension universelle. Le manuel d'Helga Bories-Sawala repose d'ailleurs sur l'idée que les Québécois ne sont pas les «cousins » des Français - le Québec ne se comprenant plus sous ce seul rapport -, mais qu'ils jouissent plutôt d'un " genre de vie nord-américain » $(D Q: 10)$, qu'ils sont dès lors partie prenante de l'Amérique et, plus spécifiquement, d'une «Amérique qui parle français », comme on le verra plus loin.

\section{Américanisation et américanité}

L'Amérique se révèle donc, à l'analyse des quatre ouvrages à l'étude, un référent polyvalent, susceptible de répondre aux besoins et aux préoccupations des différents compilateurs. Il demeure néanmoins que les ouvrages d'un même pays présentent des tendances communes quant à l'ancrage américain de la littérature québécoise. Les anthologies françaises adoptent, d'une part, une conception historique et géographique de l'Amérique comme continent vierge à découvrir et à habiter. D'autre part, les textes plus récents contenus dans Amérique, Amériques! proposent une conception idéologique de l'Amérique, laquelle est restreinte aux États-Unis perçus comme capitalistes et superficiels. En ce sens, les anthologies françaises adoptent des points de vue qui renvoient au phénomène de l'américanisation, selon deux définitions possibles: une première acception réfère précisément au «processus d'acculturation des immigrés européens entrant en contact avec le nouveau continent 》 (FENDLER ET VATTER 2004 : 60) - «S'américaniser, alors, écrit Yvan Lamonde, c'était être d'Amérique » (LAMONDE 2004: 23) -, alors qu'une acception plus contemporaine, souvent connotée négativement, désigne l'impérialisme américain et la «propagation unilatérale de modèles et de produits culturels américains » (FENDLER ET VATTER 2004 : 61), au sens métonymique d'étatsuniens dans les deux cas.

En comparaison, les ouvrages allemands - abandonnant la fascination pour les grands espaces naturels - regroupent des récits contemporains et urbains, dont l'action est principalement située à Montréal, ville cosmopolite et ouverte sur le monde, apte à figurer «les territoires de l'interculturalité américaine» (MORENCY 2013 : 120). Ce faisant, ils insistent plutôt sur le mode de vie américain ou sur l'américanité du Québec et de sa littérature. Celle-ci, partie prenante de l'identité culturelle, se définit en effet par «quelques traits qui résument les caractéristiques du monde américain, comme la jeunesse, [...] le melting pot, l'oubli de l'Europe [...] » (MORENCY $2004: 34$ ), tels qu'on les a rencontrés dans les ouvrages allemands. 


\section{LA LITTÉRATURE QUÉBÉCOISE EN TERRE D’AMÉRIQUE}

Bref, si l'américanisation désigne le processus d'acculturation - qui se traduit inévitablement par une perte ou une redéfinition des traits culturels d'origine déplorée dans les anthologies françaises -, l'américanité enregistre le résultat de ce processus, en fonction de la nouvelle identité métissée. Celle-ci, si elle peut être critiquée ${ }^{15}$, ne peut être condamnée d'emblée, puisque la conscience d'appartenance au continent américain découle de l'expérience et de la vie quotidienne. Aussi se révèle-t-elle tout autant dans la thématisation du rêve américain que, de façon plus banale, dans l'évocation de symboles comme le hockey $(D Q: 187-189)$, les bus scolaires jaunes $(D Q-U: 11)$ ou même le pâté chinois, sans lequel, aux dires de Bernard Arcand cité dans le manuel, «l'Amérique serait pas $[\mathrm{sic}]$ ce qu'elle est » $(D Q: 196)$.

\section{Le français en Amérique}

Si le Québec appartient à l'Amérique sous plusieurs aspects, il se distingue d'au moins une manière de la majorité américaine : par l'usage qui y est fait de la langue française. En ce sens, il est intéressant d'étudier la façon dont les ouvrages présentent les spécificités de la langue française en Amérique. Ce deuxième point sera cependant l'objet d'un traitement plus bref, puisque les considérations sur la langue correspondent, de manière générale, aux tendances déjà observées. Il va donc sans dire qu'il existe aussi à ce propos une distinction marquée entre les points de vue français et allemands.

\section{Les particularismes de la langue française américaine}

Du côté français, l'attention des anthologistes est surtout portée sur les particularismes de la langue française en terre d'Amérique. Les spécificités de la langue québécoise participent en cela à l'effet de pittoresque qui oriente la perception française sur le Québec. Les compilateurs d'Amérique, Amériques! justifient d'ailleurs l'intérêt de l'anthologie par le double effet de décalage linguistique que permet la juxtaposition de textes de la Nouvelle-France et d'écrits québécois contemporains : il s'agit d'ajouter «au bonheur de "faire trempette" dans la langue de Montaigne et de Malherbe, [...] le plaisir de lire des auteurs d'aujourd'hui désireux “d'enrichir le français d'une partition

\footnotetext{
${ }^{15}$ Certains textes des ouvrages allemands présentent en effet une critique d'une américanité jugée trop redevable aux États-Unis, mais celle-ci passe, le plus souvent, par l'humour, comme en témoigne l'extrait du Nez qui voque de Réjean Ducharme selon lequel « in Kanada ist nur noch der Botschafter des Planeten Mars kein Amerikaner. » (AsA : 41-42) («Au Canada, il n'y a plus que l'ambassadeur de la planète Mars qui ne soit pas américain. » (Je traduis.))
} 


\section{Sophie DUBOIS}

américaine" " (AA:14). L'anthologie met donc en scène deux variantes du français s'écartant, dans le temps comme dans l'espace, de la norme actuelle de l'ancienne métropole. De façon similaire, les directeurs de Québec-Acadie préfèrent au terme « Canada français » celui de "Canada francophone » $(Q A$ : $\mathrm{V})$, créant ainsi une distance entre le français hexagonal et celui parlé au Canada. Ce dernier se voit dès lors conférer un caractère d'étrangeté et de curiosité qui sied bien à l'exotisme privilégié dans les anthologies françaises.

Il n'est donc pas surprenant de retrouver, dans les récits regroupés dans les ouvrages (en particulier dans Québec-Acadie), nombre de transcriptions de l'oralité du parler canadien mettant en évidence ses particularités. Familiarité, contractions, jurons et québécismes abondent par exemple dans des phrases comme: «On vous attendait, mamzelle Letellier. Entrez donc! J'finis d'étendre mon lavage. Otez vot' capot. » $(Q A: 613)$ ou « Le bâtard d'enfant d'chienne ! L'écœurant, le puant... [...] Ça prend un sale restant de charogne, un baptême de constipé... » $(Q A: 939)$. La narration elle-même est ponctuée de particularismes lexicaux ou d'expressions typiques, aussitôt expliquées au lecteur (lequel est donc supposé européen et peu familier à la langue québécoise). Parmi les plus courantes se trouvent «les créatures »-en italique chez Maurice Genevoix ( $Q A: 791)$-, qui «sont, comme chacun sait, les femmes » $(Q A:$ 646), ou encore «bleuet» $(Q A: 953)$ - dont la prononciation serait « bluet» $(Q A: 575)$ selon Marie Le Franc - qui est « la luce ou myrtille en France » $(Q A: 203)$ ou «le raisin [du Québec]»( $Q A: 329)$, suivant les explications fournies dans Maria Chapdelaine et Menaud, maître draveur.

Les particularités lexicales et sémantiques de la langue québécoise sont donc décrites, expliquées et thématisées à l'intérieur même des récits. Mais c'est la prononciation - cet « accent traînant du pays du Québec » $(Q A: 784)$ - qui fait le plus souvent l'objet de commentaires : " ces mots émigrés de France depuis si longtemps, qui revêt[ent, dans la] bouche [des Canadiens,] un zézaiement plein de charme » $(Q A: 592)$ rappellent en effet à la fois l'origine et la distance de la langue québécoise. À d'autres occasions, c'est plutôt l'accent hexagonal qui est pris à parti, mais il ne faut pas s'y méprendre : il s'agit en réalité d'un nouveau moyen utilisé par les auteurs pour commenter, par la négative cette fois, l'accent du Québec. Ce passage où Maria Chapdelaine décrit le parler des Français venus acheter la terre de Lorenzo Surprenant est, à ce propos, révélateur :

Les paroles qui sortaient de leur bouche sonnaient comme des mots d'une langue étrangère. Ils n'avaient pas la lenteur de la diction 


\section{LA LITTÉRATURE QUÉBÉCOISE EN TERRE D’AMÉRIQUE}

canadienne, ni cet accent indéfinissable qui n'est pas l'accent d'une quelconque province française, mais seulement un accent paysan, en quoi les parlers différents des émigrants d'autrefois se sont confondus. $(Q A: 247)$

Enfin, si on retrouve aussi dans les anthologies françaises la présence d'un certain nombre de termes issus de la langue anglaise, ceux-ci sont simplement présentés comme des traits du français nord-américain et ils se résument à un l'usage d'un certain lexique (par exemple, dans une phrase comme : "il lui avait trouvé une place dans un "drugstore", sorte de bazar où [...][o]n débitait de l'ice-cream [et] du chewing-gum » $(Q A: 698))$. En ce sens, l'usage de l'anglais participe de façon inhérente à la part de pittoresque qui compose la langue des Canadiens français, au même titre que l'accent ou que les autres particularités sémantiques ou lexicales mises de l'avant dans les différents récits.

\section{Le français dans la polyphonie américaine}

Les ouvrages allemands qui incluent le Canada, à côté de la Suisse et de la Belgique, dans les «mehrsprachige Länder» (AsA: 7) (les pays multilingues) s'intéressent, pour leur part, davantage à la situation de cohabitation linguistique en Amérique qu'à la singularité du français québécois. Se servant de l'image des irréductibles francophones en terre nord-américaine ${ }^{16}$ ou de la métaphore de l'île francophone dans un océan anglophone ${ }^{17}$, les deux ouvrages mettent en évidence la situation linguistique (« Sprachsituation » (DQ-U: 32)) particulière - minoritaire, précaire, mais aussi ardemment défendue - du français en Amérique.

Dans le manuel sur le Québec particulièrement, les politiques linguistiques, souvent associées aux débats constitutionnels, occupent une place importante, à l'intérieur de laquelle la littérature sert d'illustration, par exemple avec la nouvelle «Oui or no" de Monique Proulx $(D Q: 67)$ qui relate le dilemme d'une femme hésitant entre son mari québécois et son amant torontois, au moment du référendum de 1995. Dans les deux ouvrages donc, comme le mentionnent Baier et Filion, la littérature est indissociable des conditions sociopolitiques du Québec comme nation francophone autonome au milieu du continent américain anglophone $(A s A: 15)$. Or, bien qu'ils contiennent certains

\footnotetext{
${ }^{16}$ « Dans cette Amérique du Nord anglophone, une poignée d'irréductibles s'obstine à parler du matin au soir la langue de Molière : les Québécois !» (DQ : 24)

${ }^{17}$ « einer frankophonen Insel in einem anglophonen Ozean » (AsA : 212).
} 


\section{Sophie DUBOIS}

propos alarmistes sur les dangers d'une folklorisation, voire d'une disparition, de la langue française en Amérique, les ouvrages envisagent aussi la situation actuelle d'une manière positive, comme une spécificité du Québec qui en fait un lieu ouvert, dynamique et diversifié, suivant le point de vue qui domine du côté allemand. D'ailleurs, selon Baier et Filion, l'isolement linguistique du Québec serait un privilège pour les auteurs québécois qui jouiraient d'une plus grande liberté créatrice que leurs collègues canadiens-anglais, lesquels ne peuvent compter sur une distinction linguistique pour établir leur différence par rapport au voisin états-unien $(A s A: 11)$.

Corollaire à la situation minoritaire du français en Amérique, la cohabitation linguistique apparaît comme une autre particularité québécoise qui trouve écho dans la littérature et qui devient manifeste en contexte américain. À ce propos, plusieurs œuvres et extraits retenus dans les deux ouvrages allemands mettent en scène et problématisent la coexistence du français et de l'anglais au Québec, qu'il s'agisse de la comédie bilingue Bon cop, bad cop traitant des clichés mutuels relevant de la perception («Wahrnemungsklichees» $(D Q-U: 49)$ ) entre Canadiens anglais et Canadiens français ou du roman Salut, Galarneau! de Jacques Godbout dont le passage « Thank you merci, Come again au revoir, Close Cover before Striking baissez la tête avant de frapper ${ }^{18}$ " fournit un nouvel exemple du recours à l'humour pour souligner les écueils de la coprésence des deux langues officielles. Dans «ce terrain mouvant où se côtoient les langues d'ici » $(D Q: 148)$ (pour citer à nouveau une nouvelle de Monique Proulx), l'anglais n'agit plus simplement comme une particularité du français canadien, mais comme une réalité qui entre en dialogue ou en conflit avec lui et qui, par là, participe de son américanité.

Il est d'ailleurs révélateur que les deux ouvrages incluent dans leurs pages des textes en anglais ou d'auteurs anglophones ${ }^{19}$, alors même que leur sous-titre ou le titre d'une de leurs sections est "Une Amérique qui parle français » ou "Québec : in Amerika französisch leben » (AsA : 287) (vivre en français en Amérique). Le français apparaît dès lors comme consubstantiel à l'anglais sur un territoire américain qui se définit par sa polyphonie; les textes anglais sont ainsi présentés comme faisant partie de la littérature québécoise, au même titre, par ailleurs, que ceux d'auteurs franco-américains, comme Antonine Maillet,

\footnotetext{
${ }^{18}$ Passage qui, dans la traduction allemande, conserve son bilinguisme : « Thank you dankeschön, Come again auf Wiedersehen, Close Cover before Striking senken Sie das Haupt » (AsA : 63).

19 Le manuel propose la chanson «No French, no more» de Zachary Richard, alors que l'anthologie contient des textes, en traduction allemande, des auteurs anglo-québécois David Homel, Trevor Ferguson, Irving Layton et Charles Taylor.
} 


\section{LA LITTÉRATURE QUÉBÉCOISE EN TERRE D’AMÉRIQUE}

Daniel Poliquin ou Zachary Richard qui trouvent place dans le manuel. Par conséquent, aborder la littérature québécoise dans une perspective continentale permet à la fois une ouverture aux autres auteurs francophones du territoire et, dans l'optique d'une dialectique linguistique, une inclusion d'auteurs anglophones écrivant au Québec. À l'inverse, il est notable que les ouvrages français regroupent presque exclusivement des auteurs d'origine québécoise voire française $\mathrm{e}^{20}$, et ce, même si les éditeurs d'Amérique, Amériques! affirment vouloir réunir " des poètes et écrivains d'expression française vivant en Amérique du Nord» $(A A: 10 \text {. Je souligne })^{21}$.

\section{Pertinence, buts et conséquences de l'ancrage américain de la littérature québécoise}

Que retenir, au final, des analyses des quatre ouvrages concernant la pertinence d'un tel ancrage américain de la littérature québécoise ? Et que nous apprend-il sur les préoccupations respectives de la France et de l'Allemagne?

Si l'inscription du Québec dans un contexte américain permet d'éviter une représentation purement nationaliste de la culture québécoise en la faisant dialoguer avec sa partie anglophone et ses branches franco-américaines, il demeure que cet ancrage n'est pas toujours convaincant ni exempt de contradictions. En effet, on vient de le voir, les anthologies françaises proposent davantage de textes français que de textes qui témoigneraient de l'existence d'une francophonie nord-américaine ou d'une littérature québécoise au fait de son appartenance contemporaine au territoire américain ${ }^{22}$. En outre, malgré la thèse énoncée par Bories-Sawala dans le manuel, le médiateur principal utilisé, dans les ouvrages allemands, pour faire le pont entre la culture québécoise et l'Allemagne reste la France, comme en témoigne la présence, au

\footnotetext{
${ }^{20}$ Outre Louis Hémon, Henri Longfellow et les rédacteurs des écrits de la Nouvelle-France, souvent inclus dans les canons québécois et franco-américain, on compte Quefellec, Le Franc, Genevoix et Constantin-Weyer dans Québec-Acadie, en plus de Patrick Dubost dans Amérique, Amériques! Dans les ouvrages allemands, seuls Jean Muzi, Yannick Jaulin et les auteurs de la bande dessinée, Achdé et Laurent Guerra, sont français et le choix de les inclure dans le manuel ne relève pas de l'auteur, mais des autorités ministérielles, puisque ce sont des œuvres obligatoires au programme.

${ }^{21}$ Il convient cependant de spécifier que, sur le plan du statut octroyé à la langue française, les textes datant de 2008 contenus dans Amérique, Amériques! se rapprochent du point de vue allemand puisqu'ils mettent en scène une Montréal «bilinguale» $(A A: 178)$ où, devant "la tentative de parler français » du narrateur de David Homel, une serveuse lui « ser[t] [s]a bière en anglais », ce qui lui fait s'exclamer : «Voilà une vraie ville ouverte !» $(A A: 198)$.

${ }^{22}$ De fait, 18 des 41 textes contenus dans ces anthologies sont d'auteurs français, alors que seuls Jean-Marc Dalpé, dramaturge franco-ontarien, et David Homel, auteur anglo-montréalais né aux États-Unis, témoignent d'un ancrage continental de l'institution littéraire québécoise.
} 


\section{Sophie DUBOIS}

programme obligatoire, de textes hexagonaux ou d'une chanson comme « Les maudits Français » de Lynda Lemay ( $D Q: 8-9)$. Par ailleurs, en dépit de la volonté des compilateurs de décrire une Amérique plurielle et autre qui se lit notamment dans les titres des ouvrages, les points de vue allemand et français n'entraînent pas une réelle redéfinition du mythe américain. L'Amérique demeure perçue, selon des représentations communes, soit comme une terre de grands espaces à explorer, soit comme une société de perdition et d'artificialité, ou encore, comme le lieu de tous les possibles et de l'American Dream.

De fait, l'ancrage américain semble servir davantage de subterfuges, d'une part, à un maintien de l'inscription de la littérature québécoise dans la filiation française, à travers l'histoire de la colonisation américaine ; d'autre part, à une ouverture vers une littérature-monde ou à une universalisation rendant pertinente la littérature québécoise aux lecteurs allemands. Considérant que « le manuel [comme l'anthologie] est un objet culturel en soi, qui nous renseigne sur la société globale dont il est issu »(LEBRUN 2007 : 2), les perspectives adoptées traduisent les préoccupations respectives des deux pays : les auteurs des ouvrages cherchent en effet - même si cela n'est pas conscient ou volontaire - à répondre à certains désirs et à certaines inquiétudes de leurs lecteurs. Aussi, en regard de l'étude des anthologies, les Français se révèlent-ils nostalgiques de leur passé colonisateur et toujours friands d'un exotisme qui place l'Autre dans une position d'étranger et de curiosité face à la norme française, qu'elle soit culturelle ou langagière. Quant aux Allemands, ils se montrent soucieux des questions liées à l'intégration des communautés culturelles et à la diversité ethnique, sociale ou linguistique, questions auxquelles ils semblent chercher, dans le cas québécois, un modèle à suivre ou, à tout le moins, à observer.

Dans cette optique, la référence américaine apparaît donc quelque peu factice, voire opportuniste, orientée autant vers une lecture de la littérature du Québec que vers les intérêts de la société d'accueil. Ces considérations pragmatiques sont particulièrement évidentes dans le cas du manuel, dans lequel BoriesSawala fait le pari que, davantage que l'image des Québécois comme « cousins » des Français, l'idée d'une présence francophone en Amérique « ein französischsprachiges Stück Amerika» (DQ-U: 6) (un morceau d'Amérique francophone) - sera plus à même de capter l'attention des étudiants allemands, déjà attirés par la culture anglo-américaine ${ }^{23}$. L'ancrage américain

23 Voir «Vorüberlegungen zum Einsatz der Textausgabe im Unterricht» (DQ-U: 6). (« Considérations sur l'utilisation du manuel en classe » (Je traduis.)). 


\section{LA LITTÉRATURE QUÉBÉCOISE EN TERRE D’AMÉRIQUE}

apparaît donc ici comme une stratégie pédagogique tablant sur la popularité d'une Amérique le plus souvent associée aux États-Unis.

Or, malgré ces quelques bémols, la principale conséquence - et le principal intérêt - de l'ancrage américain sur la perception de la littérature québécoise (et sur la vision du Québec qu'elle véhicule) est la focalisation externe, décalée qu'il permet. En effet, tout comme le mythe américain résulte d'une perception européenne, les ouvrages à l'étude abordent le Québec en partie à travers le regard de l'Autre. De fait, plus du quart des récits et morceaux choisis présente un point de vue sur le Québec émanant de l'extérieur : que ce soit celui des explorateurs arrivant en Nouvelle-France, celui des auteurs français ayant séjourné au Québec au début du $\mathrm{XX}^{\mathrm{e}}$ siècle ou, plus récemment, celui des immigrants s'installant au Canada. Dès lors, considérant la nature des ouvrages à l'étude (c'est-à-dire des anthologies et manuels de littérature étrangère), l'ancrage américain offre un point de vue externe auquel le lecteur français ou allemand se sent légitime de superposer son propre regard d'observateur découvrant le Québec. L'Amérique constitue, en définitive, un espace médian qui, en élargissant la focalisation, justifie le regard excentré porté sur la province et rend ainsi possible l'appréhension et l'appropriation de la littérature québécoise à l'étranger.

\section{Bibliographie}

\section{Corpus à l'étude}

Amérique, Amériques! Écrits du Québec, 1608/2008 (2008), édité par Michel Kneubühler et Thierry Renaud, Genouilleux, La Passe du vent ; Montréal, L'Instant même, « Haute mémoire ».

Anders schreibendes Amerika. Eine Anthologie der Literatur aus Québec 19452000 (2000), dirigé par Lothar Baier et Pierre Filion, Heidelberg, Wunderhorn Verlag.

Découvrir le Québec. Une Amérique qui parle français (2010), manuel réalisé par Helga Bories-Sawala, Paderborn, Schöningh, «EinFach Französisch» [inclut également un livre du professeur (Unterrichtsmodell) et un DVD]. 


\section{Sophie DUBOIS}

Québec-Acadie. Rêves d'Amérique (1998), romans et nouvelles choisis et présentés par Jean-Paul Bouchon, Alain Quella-Villégier et Dominique-Anne Villégier, Paris, omnibus, « Une autre façon de voyager ».

\section{Corpus théorique}

CHOPPIN, Alain (2007), «Le manuel scolaire: un objet commun, des approches plurielles », in LEBRUN, Monique (dir.), Le manuel scolaire : d'ici et d'ailleurs, d'hier à demain, Québec, Presses de l'Université du Québec, pp. 109-116.

FENDLER, Ute et VATTER, Christophe (2004), «Évangéline multimédia. Un mythe acadien entre américanité et américanisation», Globe: revue internationale d'études québécoises, vol. $7, \mathrm{n}^{\mathrm{0}} 2$, pp. 59-89.

FRAISSE, Emmanuel (1997), Les anthologies en France, Paris, Presses universitaires de France, « écriture ».

LAMONDE, Yvan (2004), "Américanité et américanisation. Essai de mise au point », Globe : revue internationale d'études québécoises, vol. 7, $\mathrm{n}^{\circ} 2$, pp. 2129.

LEBRUN, Monique (dir.) (2007), Le manuel scolaire: d'ici et d'ailleurs, d'hier à demain, Québec, Presses de l'Université du Québec.

MORENCY, Jean (1994), Le mythe américain dans les fictions d'Amérique. De Washington Irving à Jacques Poulin, Québec, Nuit blanche.

--- (2013), «Les manifestations du phénomène interculturel dans les textes littéraires : une exploration de la question de l'américanité québécoise », in ALI-KHODJA, Mourad, MERKLE, Denise, MORENCY, Jean et THÉRIAULT, Jean-François (dir.), Territoires de l'interculturalité : expériences et explorations, Québec, Presses de l'Université Laval, pp. 109121.

PAGEAUX, Daniel-Henri (1995), « Recherches sur l'imagologie : de l'Histoire culturelle à la Poétique », Thélème : Revista complutense de estudios franceses, no 8, pp. 135-160. 
LA LITTÉRATURE QUÉBÉCOISE EN TERRE D’AMÉRIQUE

--- (2007), Littérature et culture en dialogue, essais réunis, annotés et préfacés par Sobhi Habchi, Paris, L'Harmattan.

Éditions Wunderhorn,

http://www.wunderhorn.de/content/verlag/index ger.html. 